\title{
Idade materna e síndrome de Down no Nordeste do Brasil
}

\author{
Maternal age and Down syndrome \\ in Northeast Brazil
}

Fábio A. F. Gusmão 1

Eraldo J. M. Tavares 1

Lília Maria de Azevedo Moreira 1

\footnotetext{
1 Instituto de Biologia, Universidade Federal da Bahia. Rua Barão de Geremoabo 147, Salvador, $B A$ 40170-290, Brasil. lazevedo@ufba.br
}

\begin{abstract}
This study analyzes the association between advanced maternal age and increased incidence of Down syndrome in neonates, based on a population sample from the State of Bahia in Northeast Brazil. Age of the mothers of 220 Down syndrome subjects was investigated, and age distribution was compared to that of the population control group, 220 mothers of subjects without Down syndrome. The proportion of Down syndrome infants dependent on advanced maternal age was estimated at 43.6\%, thus showing a high correlation $(r=0.95)$ between advanced maternal age and Down syndrome incidence. However, this component was significantly lower than the $75 \%$ reported in the literature. The component independent of maternal age was estimated at 56.4\%, indicating the action of other factors on meiotic non-disjunction associated with 21 trisomy. The results also indicate that despite the regional characteristics of Northeast Brazil, factors both dependent and independent of maternal age show the same distribution observed in Southeast Brazil, where extensive studies have been performed.
\end{abstract}

Key words Maternal Age; Down Syndrome; Trisomy

Resumo Analisa-se, aqui, a associação entre idade materna avançada para procriação e aumento da incidência de progênie com síndrome de Down em amostra populacional do Estado da Bahia. Compararam-se as idades das mães de 220 portadores de síndrome de Down ao grupo-controle composto por mães de mesmo número de nascidos vivos, sem a síndrome de Down. Estimou-se em 43,6\% o percentual de síndrome de Down dependente da idade materna indicando uma alta correlação $(r=0,95)$ entre idade materna e síndrome de Down. Este componente foi, entretanto, significantemente menor do que o percentual de $75 \%$ referido na literatura. Estimouse o componente independente da idade materna em 56,4\%, evidenciando a importância de outros fatores, no processo de não-disjunção meiótica associada à trissomia 21. Os resultados encontrados mostram também que, não obstante as características próprias da Região Nordeste, os fatores dependentes e independentes da idade materna apresentam a mesma tendência de distribuição observada na Região Sudeste do Brasil, onde extensivos estudos têm sido realizados.

Palavras-chave Idade Materna; Síndrome de Down; Trissomia 


\section{Introdução}

A idade reprodutiva está inequivocamente relacionada à origem de trissomias cromossômicas em humanos e a idade materna avançada, comumente designada como acima de 35 anos, é uma variável fortemente associada à síndrome de Down. A freqüência desta síndrome é estimada em cerca de 1 em 600 nascidos vivos e em 150 concepções (Hernández \& Fisher, 1996). Na maioria dos casos (95\%), o cromossomo extra é derivado de não-disjunção na meiose I de origem materna (Antonarakis, 1998). Segundo dados do Estudo Colaborativo Latino-Americano de Malformações (ECLAMC), $40 \%$ dos nascidos com síndrome de Down têm mães com idades entre 40 e 44 anos, embora mulheres nesta faixa etária sejam responsáveis por apenas $2 \%$ do total de nascimentos (Castilla et al., 1995).

Penrose (1961) afirma que o aumento da taxa de síndrome de Down em progênie de mães cuja idade ultrapassa 35 anos é devido à soma de no mínimo dois componentes, um independente da idade materna e outro dependente. A trissomia pode estar relacionada a erros na formação de quiasmas e a pareamento de cromossomos meióticos, assim como ao decréscimo da seleção contra óvulos e embriões aneuplóides em mulheres idosas (Aymé \& LippmanHand, 1982; Carothers, 1993; Chandley, 1993; Zheng \& Byers, 1992).

Hassold \& Sherman (2000) referem que o efeito da idade está, entretanto, restrito aos casos de origem materna e que casos de trissomia 21 de origem paterna ou de origem pós-zigótica são independentes da idade. Os autores propõem um modelo de dois eventos (two-hit) para a não-disjunção materna. O primeiro evento, independente da idade materna, poderia estar associado a uma configuração vulnerável de quiasmas. O segundo evento, dependente da idade, compreenderia o processamento anormal de um bivalente vulnerável na meiose I. De acordo com esta interpretação, em mães jovens ou idosas pode ocorrer o mesmo processo de não-disjunção; simplesmente ocorre mais freqüentemente com a idade, possivelmente devido à degradação do processo meiótico associado à idade.

A ocorrência de síndrome de Down independente da idade pode estar ainda relacionada à mutação de genes que atuam no processo de disjunção meiótica. James et al. (1999) referem que o metabolismo do folato em mães de crianças com síndrome de Down é anormal e sugerem que uma mutação no gene da 5,10metilenotetrahidrofolato redutase (MTHFR), que regula as reações celulares de metilação poderia levar à hipometilação e, em conseqüência, a erros na segregação cromossômica. Da Silva et al. (2002) estudaram os genótipos dos polimorfismos dos genes MTHFR, MTR, MTRR e CBS em 150 mães de crianças com síndrome de Down e controles, considerando a mutação C677T do gene MTHFR como um fator de risco para ocorrência do distúrbio. Shonn et al. (2000) também destacam como importante fator de risco a ocorrência da mutação de gene MAD 2, essencial à associação das cromátides aos microtúbulos do fuso mitótico.

Comparações entre freqüências de casos dependentes da idade em diferentes estudos mostram resultados muitas vezes conflitantes, associados a variáveis demográficas que influem na incidência de transtornos cromossômicos, devido a sua relação com a idade materna (McGrother \& Marshall, 1990). Populações em que as mulheres tem filhos até o final da sua vida reprodutiva mostram a incidência de nascimentos de crianças com transtornos cromossômicos de até 6/1.000 e a proporção de nascidos vivos com a síndrome de Down em aproximadamente 3/1.000. Dados da Organização Mundial da Saúde (OMS) referentes a países europeus mostram que apenas a ação eugênica do planejamento familiar foi responsável pela redução de 30 a $60 \%$ na incidência da síndrome de Down (Modell et al., 1992).

Dados da Fundação Instituto Brasileiro de Geografia e Estatística (IBGE) mostram que, no Brasil, as mulheres entre 20 e 34 anos representavam, em 1970, 68,6\% da taxa total de fecundidade, elevando-se para $71 \%$ em 1971, e 10,5\% em 1984, tendência acompanhada de queda nas idades mais avançadas, mostrando, assim uma mudança de estrutura típica de países de alta fecundidade para os de baixa fecundidade. Observa-se, em todas as regiões, uma perda de representatividade da fecundidade nos grupos etário acima de 35 anos, variando de intensidade a depender da região, mostrando no Nordeste baixa menos acentuada. (Simões, 1988).

Utilizando modelos matemáticos analisase, aqui, a relação entre a idade materna e a freqüência de nascimentos de crianças com síndrome de Down no Nordeste do Brasil, onde faltam estatísticas sobre a incidência da síndrome e a proporção de casos independentes e dependentes da idade materna. 


\section{Métodos}

A amostra de portadores de síndrome de Down analisados neste trabalho foi constituída por 220 casos atendidos no Serviço de Genética/Laboratório de Genética Humana e Citogenética do Instituto de Biologia da Universidade Federal da Bahia (UFBA), entre 1994 e 1997, no programa de extensão Genética na Prática Social para registro de casos e orientação genética. Como grupo controle, tomou-se uma amostra casual de crianças nascidas no mesmo período em uma maternidade pública de Salvador, Bahia, que atende a $20 \%$ dos nascimentos ocorridos nessa cidade. Selecionaram-se aleatoriamente os casos, listando os prontuários de crianças sem síndrome de Down e, retirando, por sorteio simples, o número de casos correspondente ao de crianças síndrome de Down atendidas no período. Incluíram-se, entre os nascimentos, apenas os conceptos com mais de $500 \mathrm{~g}$, de acordo com as recomendações do Centro Latino-Americano de Perinatologia e Desenvolvimento Humano (CLAP) - que classificam os fetos com $500 \mathrm{~g}$ ou menos como abortos, mesmo que tenham sido expulsos ou extraídos com sinais de vida (Belitzky et al., 1978).

A análise dos fatores dependentes e independentes da idade materna foi feita de acordo com Beiguelman et al. (1996). O tratamento estatístico foi feito de acordo com este autor e Lamson \& Hook (1981). As médias das idades maternas foram comparadas não-parametricamente com o teste $t$ de Student e as diferenças analisadas com o teste do qui-quadrado $\left(\chi^{2}\right)$. O nível de significância adotado para todas as comparações foi de $5 \%$.

\section{Resultados}

Na Tabela 1, pode-se observar a distribuição das crianças com síndrome de Down e o grupo-controle, em função da idade materna. $\mathrm{Na}$ Figura 1, representa-se esta distribuição. Verifica-se que a idade das mães com filhos com síndrome de Down é significativamente mais elevada do que a de mães com filhos normais, demonstração feita a partir da análise não paramétrica e pela distribuição dos dados da Tabela $1\left(\chi^{2}=431,24 ; \mathrm{GL}=9 ; \mathrm{p}<0,001\right)$.

Da mesma forma que na amostra de Beiguelman et al. (1996), a distribuição gráfica mostra curva sugestiva de distribuição bimodal com o primeiro pico referente à idade materna de 25 a 29 anos e o segundo na faixa de 35 a 39 anos. A média de idade das mães de filhos com síndrome de Down $(33,02 \pm 18,51)$ foi sig-

\begin{tabular}{|c|c|c|}
\hline \multicolumn{3}{|c|}{$\begin{array}{l}\text { Distribuição percentual da síndrome de Down e de crianças normais } \\
\text { de acordo com a idade materna. }\end{array}$} \\
\hline Idade materna (anos) & $\begin{array}{l}\text { Síndrome de Down } \\
\qquad(n=220)\end{array}$ & $\begin{array}{l}\text { Controle } \\
(n=220)\end{array}$ \\
\hline$\leq 15$ & - & 5,9 \\
\hline $15-19$ & 5,0 & 21,8 \\
\hline $20-24$ & 11,0 & 28,9 \\
\hline $25-29$ & 20,0 & 21,5 \\
\hline $30-34$ & 15,5 & 10,0 \\
\hline $35-39$ & 25,6 & 10,5 \\
\hline $40-44$ & 19,0 & 0,90 \\
\hline $45-49$ & 3,0 & 0,50 \\
\hline $50-54$ & 0,45 & - \\
\hline $55-59$ & 0,45 & - \\
\hline Média & 33,02 & 24,4 \\
\hline Desvio-padrão & 18,51 & 3,83 \\
\hline
\end{tabular}

$\chi^{2}=431,24 ; G L=9 ; P<0,001$.

Figura 1

Distribuição da idade materna percentual nos dois grupos estudados.

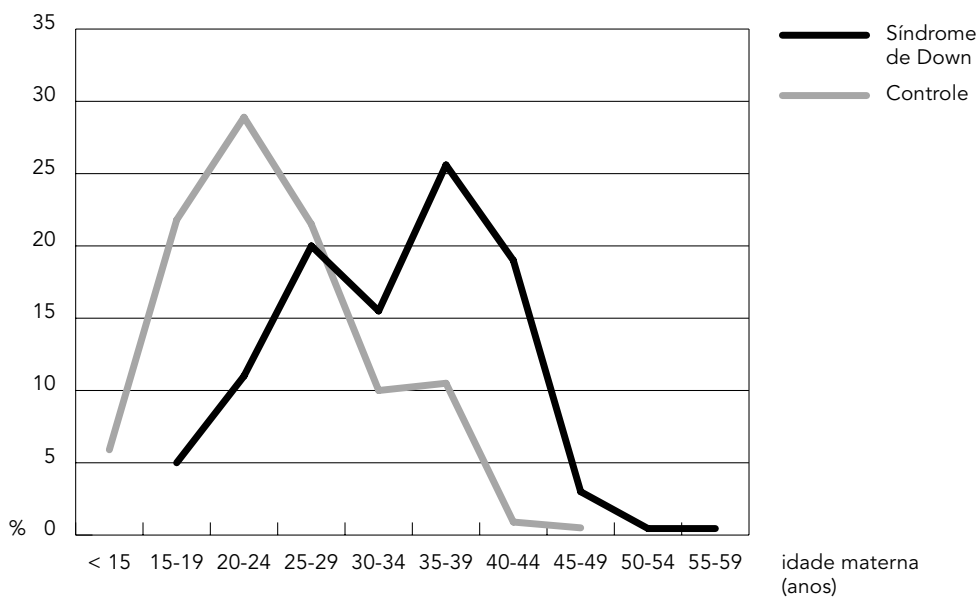

nificativamente superior $(\mathrm{t}=5,3 ; \mathrm{p}<0,05)$ à das mães do grupo controle $(24,4 \pm 3,83)$.

Estimou-se que, entre as crianças com síndrome de Down, o componente independente da idade materna apresentou decréscimo de $20 \%$ para $9,3 \%$. O mesmo procedimento aplicado aos intervalos subseqüentes permitiu a preparação da Tabela 2, na qual se estabeleceu 
a probabilidade de independência da idade materna.

A Figura 2, podem-se observar as estimativas contidas na Tabela 2 . O somatório destas estimativas mostra que o componente independente da idade materna explica $56,4 \%$ dos casos, enquanto que o componente dependente da idade materna foi de $43,6 \%$, para as crianças com síndrome de Down, nascidas na região metropolitana de Salvador, com diferenças estatisticamente significativas $\left(\chi^{2}=3,27\right.$; GL $=1$; $\mathrm{p}<0,10)$.

Tabela 2

Distribuição da idade materna dependente e independente da síndrome de Down.

\begin{tabular}{lccc}
\hline $\begin{array}{l}\text { Idade materna } \\
\text { (anos) }\end{array}$ & $\begin{array}{c}\text { Idade- } \\
\text { independente }\end{array}$ & $\begin{array}{c}\text { Idade- } \\
\text { dependente }\end{array}$ & $\begin{array}{c}\text { Probabilidade da } \\
\text { idade-independente }\end{array}$ \\
\hline $15-19$ & 5,0 & - & $\sim 1$ \\
$20-24$ & 11,0 & - & $\sim 1$ \\
$25-29$ & 20,0 & - & $\sim 1$ \\
$30-34$ & 9,3 & 6,2 & 0,6 \\
$35-39$ & 9,8 & 15,8 & 0,38 \\
$40-44$ & 0,8 & 18,2 & 0,04 \\
$45-49$ & 0,5 & 2,5 & 0,16 \\
$50-54$ & - & 0,45 & $\sim 0$ \\
$55-59$ & - & 0,45 & $\sim 0$ \\
\hline
\end{tabular}

Figura 2

Percentual estimado da idade materna dependente e independente da síndrome de Down.

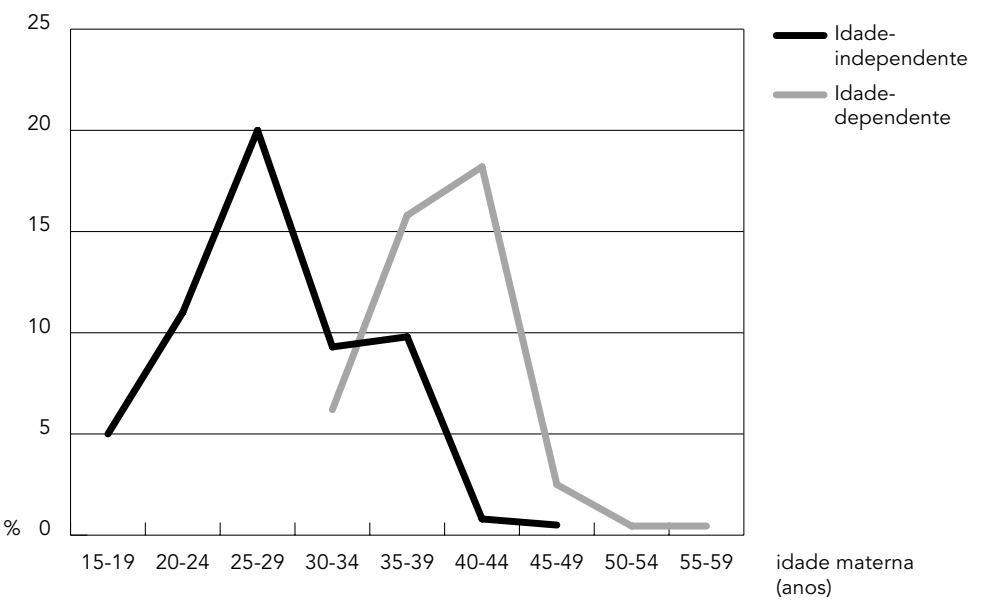

Cad. Saúde Pública, Rio de Janeiro, 19(4):973-978, jul-ago, 2003
Na Tabela 3 e na Figura 3, apresentam-se percentuais comparativos da distribuição de crianças com síndrome de Down de Salvador (Bahia) e Campinas (São Paulo). A análise nãoparamétrica evidencia que a média de idade das mães de filhos com síndrome de Down nascidos em Salvador $(33,02 \pm 18,51)$ também não difere significativamente $(\mathrm{t}=0,17 ; \mathrm{p}<0,05)$ da das mães de Campinas $(33,34 \pm 9,73)$. A prevalência estimada entre os grupos dependentes e independentes da idade materna não mostra diferenças estaticamente significativas entre a presente amostra e o grupo populacional do Sudeste, como se pode ver na Figura 3.

\section{Discussão}

A distribuição etária das mães com filhos com síndrome de Down (Figura 1) indica a ocorrência de fatores dependentes e independentes da idade materna. Calculou-se o percentual de síndrome de Down dependente e independente da idade materna em função desta distribuição, a partir do primeiro pico, correspondente ao intervalo da idade materna entre 25-29 anos (20\%), em referência a freqüência de nascimentos crianças controle $(21,5 \%)$ nessa faixa etária. No intervalo de idade materna seguinte, de 30 a 34 anos, a freqüência de nascimentos no grupo-controle diminui para $10 \%$.

As diferenças são semelhantes às encontradas por Beiguelman et al. (1996) para a Região Sudeste do Brasil e mostram que o componente dependente da idade materna não apenas é significativamente menor do que o percentual de $75 \%$ estimado por Penrose (1961), mas também inferior aos valores referidos por Lamson \& Hook (1980), que variam de 59,6\%, para Nova York, a 49,3\%, para amostra da Suécia.

Da mesma forma que no estudo de Beiguelman et al. (1996), as presentes diferenças foram também explicadas pela composição mais jovem da população presentemente analisada, com média de 24,4 anos no grupo-controle, em relação à idade materna da população geral, calculada pelo referidos autores a partir dos dados de Hook \& Chambers (1977) em 26,1 para Nova York, 27,01 para Massachusetts (Hook \& Fabia, 1978), 25,88 para Suécia (Hook \& Lindsjo, 1978), 25,98 para Austrália (Sutherland et al., 1979), assim como 24,77 para a cidade de Campinas, São Paulo, Brasil (Beiguelman et al., 1996). Na amostra de Salvador, Bahia, Brasil, a média de idade materna foi de 24,4 anos e observou-se correlação alta $(r=0,95)$ entre o componente dependente da idade materna nos pa- 
cientes com síndrome de Down quando comparados à população geral.

Do ponto de vista populacional, os resultados obtidos indicam que as distribuições das faixas de natalidade modificaram-se significativamente desde 1950, quando comparadas aos dos dados censitários analisados por Martello \& Frota-Pessoa (1969). De acordo com dados do Sistema de Informação sobre Nascidos Vivos (SINASC) do Ministério da Saúde, referentes ao período de 1994 a 1997, no Norte e Nordeste brasileiro nasce maior número de crianças de mães com idade superior a 35 anos, colocando, assim, o Nordeste como uma região de maior risco para a síndrome de Down em relação à Região Sudeste (DATASUS, 2002).

Entretanto, não obstante as diferentes características demográficas dessas regiões, o componente síndrome de Down dependente da idade materna encontrado no presente estudo foi da mesma ordem que o verificado na Região Sudeste do Brasil e inferior ao encontrado para populações não-brasileiras, não confirmando assim, uma maior expectativa de nascimentos de crianças com síndrome de Down na Região Nordeste do Brasil.

\begin{tabular}{|c|c|c|}
\hline \multicolumn{3}{|c|}{$\begin{array}{l}\text { Comparação da distribuição em percentual de crianças com síndrome } \\
\text { de Down de acordo com a idade materna. }\end{array}$} \\
\hline \multirow[t]{2}{*}{ Idade materna (anos) } & \multicolumn{2}{|c|}{ Síndrome de Down } \\
\hline & Salvador $(n=220)$ & Campinas, $1996(n=200)$ \\
\hline$<15$ & - & - \\
\hline $15-18$ & 0,9 & 4,5 \\
\hline $18-21$ & 5,5 & 8,0 \\
\hline $21-24$ & 7,0 & 9,5 \\
\hline $24-27$ & 10,0 & 12,5 \\
\hline $27-30$ & 13,0 & 11,0 \\
\hline $30-33$ & 10,5 & 10,0 \\
\hline $33-36$ & 9,0 & 10,5 \\
\hline $36-39$ & 16,0 & 13,5 \\
\hline $39-42$ & 15,0 & 11,0 \\
\hline $42-45$ & 8,5 & 5,5 \\
\hline $45-48$ & 2,3 & 3,5 \\
\hline $48-51$ & 0,9 & - \\
\hline $51-54$ & 0,5 & 0,5 \\
\hline $54-57$ & 0,9 & - \\
\hline Média & 33,51 & 31,34 \\
\hline Desvio-padrão & 11,02 & 9,73 \\
\hline
\end{tabular}

Figura 3

Crianças com síndrome de Down de acordo com a idade materna em populações das regiões

Nordeste e Sudeste do Brasil.

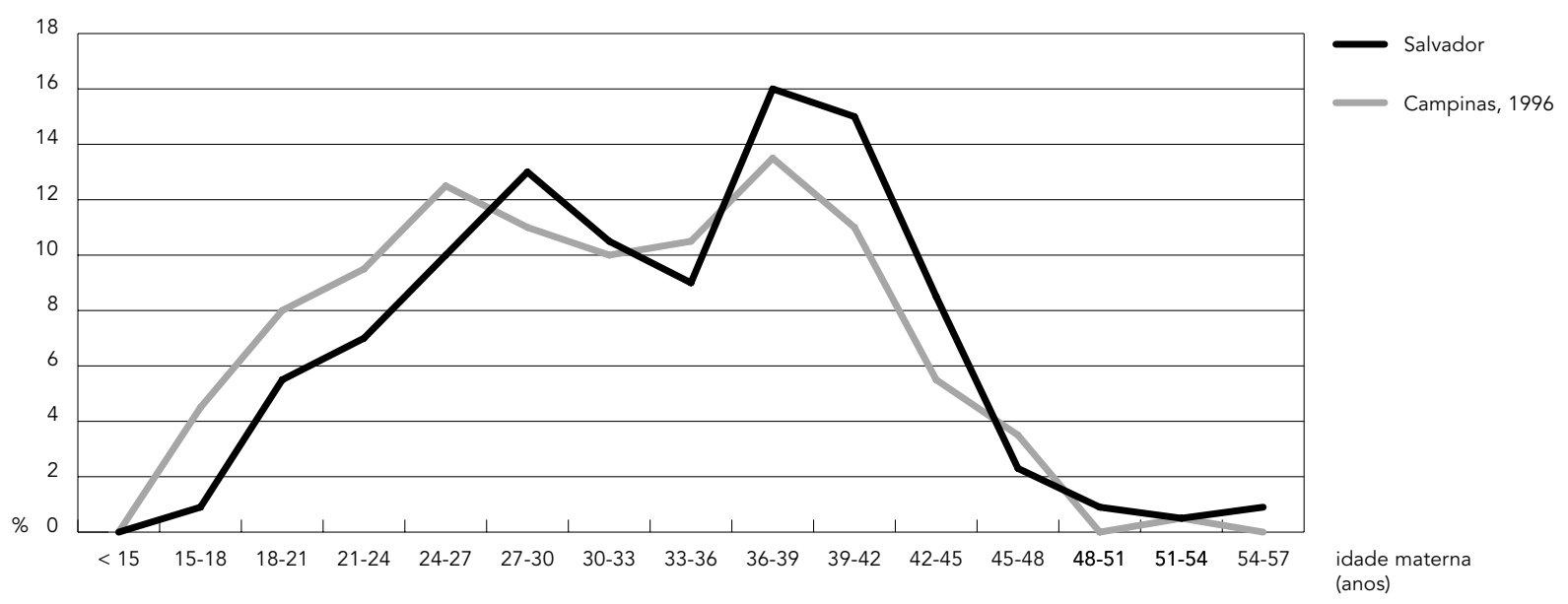




\section{Referências}

ANTONARAKIS, S. E., 1998. 10 years of genomics, chromosome 21, and Down syndrome. Genomics, 51:1-16.

AYMÉ, S. \& LIPPMAN-HAND, A., 1982. Maternal-age effect in aneuploidy: Does altered embrionic selection play a role? American Journal of Human Genetics, 34:558-565.

BEIGUELMAN, B.; KRIEGER, H. \& SILVA, L. M., 1996. Maternal age and Down syndrome in Southeastern Brazil. Revista Brasileira de Genética, 19: 637-640.

BELITZKY, R.; FESCINA, R. E. \& UCIEDA, F., 1978. Definiciones y terminología aplicables al periodo perinatal. Recomendaciones de la Organización Mundial de la Salud y modificaciones de la FIGO. Publicación Científica del CLAP, 757:136-147.

CAROTHERS, A. D., 1993. Oocyte selection model for the maternal age-dependency in Down syndrome. Human Genetics, 92:642.

CASTILLA, E. E.; LOPEZ-CAMELO, J. S. \& PAZ, J. E., 1995. Atlas de las Malformaciones Congénitas en Sudamérica. Rio de Janeiro: Editora Fiocruz.

CHANDLEY, A. C., 1993. The relationship between meiotic chromosome pairing and chiasma formation. Human Genetics, 92:643.

DA SILVA, L. R. J.; VERGANI, N.; BRUNONI, D.; LONGHITANO, S. B.; GALDERI, L.; PORTO, M. R.; D'ALMEIDA, V. \& PEREZ, A. B. A., 2002. Genes envolvidos no metabolismo da homocisteína e sua relação com risco aumentado para Síndrome de Down. In: 14o Congresso Brasileiro de Genética Clínica, Anais, p. 21, Ribeirão Preto: Sociedade Brasileira de Genética Clínica.

DATASUS (Departamento de Informática do SUS), 2002. SINASC - Sistema de Informação sobre Nascidos Vivos. 1 Agosto 2002 <http:/ / www.datasus. gov.br>.

HASSOLD, T. \& SHERMAN, S., 2000. Down syndrome: Genetic recombination and the origin of the extra chromosome 21. Clinical Genetics, 57:95-100.

HERNANDEZ, D. \& FISHER, E. M. C., 1996. Down syndrome genetics: Unravelling a multifactorial disorder. Human Molecular Genetics, 5:1411-1416.

HOOK, E. B. \& CHAMBERS, G. M., 1977. Estimated rates of Down syndrome in live births by one year maternal age intervals for mothers aged 20-49 in a New York State study - Implications of the risk figures for genetic conseling and cost-benefit analysis of prenatal diagnosis programs. Birth Defects, 13:123-141.

HOOK, E. B. \& FABIA, J. J., 1978. Frequency of Down syndrome in livebirths by single-year maternal age interval: Results of Massachusetts study. Teratology, 17:223-228.

HOOK, E. B. \& LINDSJÖ, A., 1978. Down syndrome in live births by single year maternal age interval in a Swedish study: Comparison with results from a New York State study. American Journal of Human Genetics, 30:19-27.
JAMES, S. J.; POGRIBNA, M.; POGRIBNY, I. P.; MELNYK, S.; HINE, J. B.; GIBSON, J. B.; YI, P.; TAFOYA, D. \& SWENSON, D. H., 1999. Abnormal folate metabolism and mutation in the methylenetetrahydrofolate reductase gene may be maternal risk factors for Down syndrome. American Journal of Clinical Nutrition, 70:495-501.

LAMSON, S. H. \& HOOK, E. B., 1980. A simple function for maternal-age-specific rates of Down syndrome in the 20-to-49-year age range and its biological implications. American Journal of Human Genetics, 32:743-753.

LAMSON, S. H. \& HOOK, E. B., 1981. Comparison of mathematical models for the maternal age dependence of Down's syndrome rates. Human Genetics, 59:232-234.

MARTELLO, N. \& FROTA-PESSOA, O., 1969. Estimativas das Freqüências ao Nascer, de Crianças Afetadas pelo Mongolismo em Populações Brasileiras. Estudos Demográficos. Rio de Janeiro: Fundação Instituto Brasileiro de Geografia e Estatística.

McGROTHER, C. W. \& MARSHALL, B., 1990. Recent trends in incidence, morbidity and survival in Down's syndrome. Journal of Mental Deficiency Research, 34:49-57.

MODELL, B.; KULLIEV, A. M. \& WAGNER, M., 1992. Community Genetics Services in Europe. WHO Regional Publication, European Series 38. Copenhagen: World Health Organization.

PENROSE, L. S., 1961. Parental age and non-disjunction. In: Human Chromosomal Abnormalities (W. M. Davidson \& D. Robertson Smith, ed.), pp. 116122, London: Staples Press.

SHONN, M. A.; McCARROL, R. \& MURRAY, A. W., 2000. Requirement of the spindle checkpoint for proper chromosome segregation in budding yeast meiosis. Science, 289:300-303.

SIMÕES, C. C. S., 1988. Perfil Estatístico de Crianças e Mães no Brasil: A Situação da Fecundidade; Determinantes Gerais e Características da Transição Recente. Rio de Janeiro: Departamento de Estatísticas e Indicadores Sociais, Fundação Instituto Brasileiro de Geografia e Estatística.

SUTHERLAND, G. R.; CLISBY, S. R.; BLOOR, G. \& CARTER, R. F., 1979. Down's syndrome in South Australia. Medical Journal of Australia, 2:58-61.

ZHENG, C. \& BYERS, B. J., 1992. Oocyte selection: A new model for the maternal age-dependence of Down syndrome. Human Genetics, 90:1-6.

Recebido em 5 de dezembro de 2001

Versão final reapresentada em 17 de setembro de 2002 Aprovado em 6 de fevereiro de 2003 